\title{
Dasatinib (BMS-35482) potentiates the activity of gemcitabine and docetaxel in uterine leiomyosarcoma cell lines
}

\author{
Micael Lopez-Acevedo ${ }^{1,5^{*}}$, Lisa Grace ${ }^{1}$, Deanna Teoh ${ }^{2}$, Regina Whitaker ${ }^{1}$, David J Adams ${ }^{3}$, Jingquan Jia ${ }^{4}$,
} Andrew B Nixon ${ }^{3}$ and Angeles Alvarez Secord ${ }^{1}$

\begin{abstract}
Background: To explore the activity of dasatinib alone and in combination with gemcitabine and docetaxel in uterine leiomyosarcoma (ULMS) cell lines, and determine if dasatinib inhibits the SRC pathway.

Methods: SK-UT-1 and SK-UT-1B uLMS cells were treated with gemcitabine, docetaxel and dasatinib individually and in combination. SRC and paxcillin protein expression were determined pre- and post-dasatinib treatment using Meso Scale Discovery (MSD) multi-array immunogenicity assay. Dose-response curves were constructed and the coefficient of drug interaction (CDI) and combination index (Cl) for drug interaction calculated.

Results: Activated phosphorylated levels of SRC and paxillin were decreased after treatment with dasatinib in both cell lines $(p<0.001)$. The addition of a minimally active concentration of dasatinib $\left(I C_{25}\right)$ decreased the $I C_{50}$ of each cytotoxic agent by 2-4 fold. The combination of gemcitabine-docetaxel yielded a synergistic effect in SK-UT-1 $(\mathrm{Cl}=0.59)$ and an antagonistic effect in SK-UT-1B $(\mathrm{Cl}=1.36)$. Dasatinib combined with gemcitabine or docetaxel revealed a synergistic anti-tumor effect $(\mathrm{CDI}<1)$ in both cell lines. The triple drug combination and sequencing revealed conflicting results with a synergistic effect in SK-UT-1B and antagonistic in SK-UT-1.

Conclusion: Dasatinib inhibits the SRC pathway and yields a synergistic effect with the two-drug combination with either gemcitabine or docetaxel. The value of adding dasatinib to gemcitabine and docetaxel in a triple drug combination is uncertain, but may be beneficial in select uLMS cell lines. Based on our pre-clinical data and known activity of gemcitabine and docetaxel, further evaluation of dasatinib in combination with these agents for the treatment of ULMS is warranted.
\end{abstract}

Keywords: Dasatinib, Leiomyosarcoma, SRC pathway, Targeted agents, Uterine sarcoma

\section{Background}

Leiomyosarcomas (LMS) are a rare and aggressive type of uterine malignancy that has an extremely poor prognosis. Uterine sarcomas represent only 3-9\% of all uterine malignancies and LMS account for $40 \%$ of all uterine sarcomas [1,2]. Even in the setting of early-stage disease 53 to $71 \%$ of women will develop recurrences that are often extra-pelvic and incurable. The prognosis is dismal with a historical progression-free survival rate at 2 years

\footnotetext{
*Correspondence: micael.lopez@duke.edu

'Division of Gynecologic Oncology, Duke Cancer Institute, Durham, NC 27710, USA

${ }^{5}$ DUMC 3079, Gynecologic Oncology, Duke University Medical Center, Durham, NC 27710, USA

Full list of author information is available at the end of the article
}

$\left(\mathrm{PFS}_{2}\right.$ yrs $)$ of only $30 \%$ for patients treated with surgery alone [3].

Chemotherapy with a combination of gemcitabine and docetaxel has shown the most promise to date. The overall response rate has ranged from $42 \%$ to $53 \%$ with a median duration of response of greater than 7 months in women with unresectable LMS [4]. Most recently, adjuvant gemcitabine and docetaxel was evaluated in women with completely resected stage I to IV uterine LMS. The $\mathrm{PFS}_{2}$ yrs was $45 \%$ with a median PFS of 13 months and the median survival was not yet reached [3]. Despite these modest improvements, there is an urgent need for innovative therapeutic approaches.

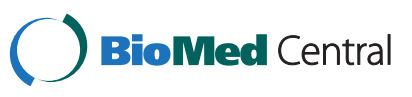


One novel and promising therapeutic agent is dasatinib (BMS-354825) (NSC 732517). Dasatinib is a potent, orally-bioavailable, small molecule inhibitor that has been shown to inhibit at least five protein tyrosine kinases/kinase families: SRC family kinases, BCR-ABL, c-KIT, EPHA2 and the PDGF $\beta$ receptor $[5,6]$. SRC kinase interacts with a variety of receptor tyrosine kinases such as EGFR, PDGFR, and c-KIT as well as other cellular factors such as focal adhesion kinase (FAK). These pathways are integral components of cellular function and regulate cellular migration, proliferation, survival, angiogenesis, and metastasis. The SRC pathway and its substrates (FAK) as well as c-KIT, EGFR and PDGF tyrosine kinase receptors have been found to be overexpressed in a wide variety of sarcomas including LMS [7-9]. Most recently, Shank et al. showed that EGFR, VEGF and c-KIT is expressed in uterine LMS specimens [9]. In particular, 57\% of uterine LMS specimens express c-KIT.

Given the activity of gemcitabine and docetaxel as well as the pre-clinical data regarding the SRC pathway in LMS, we sought to explore the activity of dasatinib (a SRC inhibitor) in combination with these cytotoxic agents in uLMS.

\section{Methods}

\section{Drugs}

Gemcitabine and docetaxel were purchased from Sigma (St. Louis, MO). Dasatinib (BMS-354825) was provided by Bristol-Myers-Squibb (Princeton, NJ) via the National Cancer Institute (NCI). Dasatinib, gemcitabine and docetaxel were dissolved in dimethylsulfoxide (DMSO). Concentrated stock solutions of all drugs were stored at $-25^{\circ} \mathrm{C}$.

\section{Cell culture}

SK-UT-1 and SK-UT-1B cell lines were obtained from the American Type Culture Collection (Manassas, VA). SK-UT-1 and SK-UT-1B cell lines are uterine in origin. Cell lines were grown and maintained in monolayer culture in RPMI 1640 (Gibco) media supplemented with $10 \%$ fetal bovine serum (Hyclone, Logan UT), $1 \%$ sodium pyruvate, 100 units $/ \mathrm{ml}$ penicillin, $100 \mathrm{ug} / \mathrm{ml}$ stremptomycin and $1 \%$ nonessential amino acids in a humidified chamber containing $5 \% \mathrm{CO}_{2}$.

\section{Meso scale discovery (MSD) analysis}

MSD analysis was performed for SRC and paxillin. Paxillin is a SRC pathway substrate that is phosphorylated by SRC and FAK upon integrin binding or growth factor stimulation. Cells were seeded at $3 \times 10^{6}$ cells per plate of each cell line, and allowed to reach confluence over 24 hours. Cells were incubated at $37^{\circ} \mathrm{C}$ for 24 hours with dasatinib at a escalating concentrations of 30, 100 and $500 \mathrm{nmol} / \mathrm{L}$. Controls were treated with DMSO. Anti-total Src antibody (tSrc) (Cell Signaling Technology, Inc.,
Danvers, MA, Cat\#2108), anti-pSrc pY418 antibody (Invitrogen, Carlsbad, CA, Cat\# 44660G), anti-total paxillin antibody (Cell Signaling Technology, Inc., Danvers, MA, Cat\#2542), or anti-phospho paxillin (Tyr118) antibody (Cell Signaling Technology, Inc., Danvers, MA, Cat\#2541) were added at $1 \mathrm{ug} / \mathrm{ml}$ to bare, goat anti-mouse plates (MSD, Gaithersburg MD), and incubated at room temperature (R.T.) for 1 hour. The plates were washed with TBS/ $0.05 \%$ Tween-20 three times and protein lysate from SK-UT-1 (20 ug total protein) or SK-UT-1B (20 ug total protein) cells were added and incubated for 2 hours at R.T. Sulfo-TAG (MSD, Cat\#R91AN-1) labeled anti-Src antibody (R\&D, Cat\# AF3389) were then added to the plates and incubated for 1 hour at room temperature after plates were washed. The plates were imaged and analyzed using a MSD Sector Imager 2400 and associated software. The electroluminescence value was normalized to each control and plotted as a percent of control. Statistical analysis was performed using two-tailed unpaired $t$-test.

\section{Cell proliferation assay}

Tumor cells were seeded at a density of 2,500 cells/well in a 96-well plate, and allowed to reach $70 \%$ confluence over 24 hours. Cells were then incubated with each drug at $37^{\circ} \mathrm{C}$ for 72 hours with escalating doses: docetaxel $(0.1 \mathrm{nmol} /$ $\mathrm{L}-1000 \mathrm{nmol} / \mathrm{L})$, gemcitabine $(0.1 \mathrm{nmol} / \mathrm{L}-100 \mathrm{nmol} / \mathrm{L})$ and dasatinib (10 nmol-4000 nmol/L). Control wells contained RPMI media only. All experiments were done using exponentially proliferating cells. After the 72 hours drug incubation period, $5 \mu$ of ATP luminescence solution was added to each well and cell proliferation was measured by ATP content using the Luminescence ATP cell detection assay system according to manufacturer's recommendations. Experiments to determine the $\mathrm{IC}_{50}$ were performed in triplicate.

The percentage of growth inhibition was calculated using

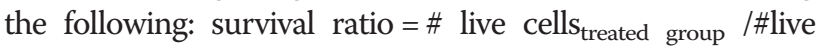
cells $s_{\text {control group }} \times 100$. The half maximal inhibitory concentration $\left(\mathrm{IC}_{50}\right)$ was defined as the drug concentration at which the $50 \%$ of the cell growth was inhibited and was analyzed using GraphPad Prism software (version 4.03 San Diego, CA). Single-agent dose response curves were constructed and the $\mathrm{IC}_{50}$ for gemcitabine and docetaxel was computed from the best fitting transition functions (determined by F-statistic). The average $\mathrm{IC}_{50}$ of all experiments performed was chosen as the final $\mathrm{IC}_{50}$. Given the lack of significant activity of dasatinib as a single-agent, the $\mathrm{IC}_{25}$ was calculated from the dose response curve. The cells were subsequently treated with combinations of gemcitabine and docetaxel; dasatinib and gemcitabine; dasatinib and docetaxel; and the three-drug simultaneous and sequential combination of gemcitabine, docetaxel, and dasatinib. For gemcitabine and docetaxel, fixed-ratio molar concentrations ranging 
from 0.125 to 4 multiples of the single-drug $\mathrm{IC}_{50}$ was used. Dasatinib was added at a fixed 1:1 ratio using the $\mathrm{IC}_{25}$.

\section{Two-drug combination effect evaluation and statistical methodologies}

The $\mathrm{IC}_{50}$ obtained for single-agent gemcitabine and docetaxel was compared to the $\mathrm{IC}_{50}$ calculated for each cytotoxic agent after adding dasatinib $\mathrm{IC}_{25}$. To analyze the drug interaction between both cytotoxic agents and dasatinib combined with either agent, the coefficient of drug interaction (CDI) was calculated. CDI is defined by the following formula; $\mathrm{CDI}=\mathrm{AB} /(\mathrm{A} \times \mathrm{B})[10,11]$. According to the absorbance of the luminescence of each group, $A B$ is the ratio of the two-drug combination group to the control group, and A or B is the ratio of the single drug group to the control group. $\mathrm{CDI}<1$ indicates synergism, $\quad \mathrm{CDI}<0.7$ significant synergism, $\mathrm{CDI}=1$ additivity and $\mathrm{CD}>1$ antagonism [11]. Due to the lack of significant activity of dasatinib and the inability to obtain an $\mathrm{IC}_{50}$ for this agent, the CDI formula was utilized to evaluate the anti-proliferative effect between dasatinib and each cytotoxic agent. This formula permits calculation of drug-to-drug interaction without a requisite $\mathrm{IC}_{50}$. All experiments were performed in duplicate.

\section{Triple drug simultaneous and sequential combination effect evaluation and statistical methodologies}

To evaluate the growth inhibition effect of dasatinib with gemcitabine and docetaxel in a triple-drug combination, we used the median effect method, which takes into account the potency of each drug combination and the shape of the dose-response curve $[12,13]$. Composite dose response curves were obtained from three independent experiments and the median effective dose, Dm (equivalent to the $\mathrm{IC}_{50}$ ) was computed using CalcuSyn software (Biosoft, Cambridge, UK). Drug interaction was assessed by the combination index (CI) method of Chou and Talalay [12,13]:

$$
\begin{aligned}
\mathrm{CI}= & (\mathrm{D}) \mathrm{A} /(\mathrm{Dx}) \mathrm{A}+(\mathrm{D}) \mathrm{B} /(\mathrm{Dx}) \mathrm{B} \\
& +\alpha(\mathrm{D}) \mathrm{A}(\mathrm{D}) \mathrm{B} /(\mathrm{Dx}) \mathrm{A}(\mathrm{Dx}) \mathrm{B}
\end{aligned}
$$

where $\mathrm{D}$ is the dose that yields $\mathrm{x} \%$ growth inhibition and $\alpha=0$ for mutually exclusive drugs (the drugs have similar sites of action). Combination index scale was defined as: $\mathrm{CI}<0.9$ synergistic, $\mathrm{CI}=0.9-1.1$ additive, $\mathrm{CI}=1.1-1.2$ slight antagonism, $\mathrm{CI}=1.2-1.45$ moderate antagonism, $\mathrm{CI}=1.45-3.3$ antagonism, $\mathrm{CI}=3.3-10$ strong antagonism.

In contrast to the two-drug combination where the CDI formula was used, the median effect (CI formula) method was utilized to evaluate triple-drug interaction. This was possible because both gemcitabine and docetaxel reached an $\mathrm{IC}_{50}$ as single agents. For sequencing studies, drug exposures were separated by 24 hours. The combination index between gemcitabine and docetaxel was computed with and without the addition of dasatinib $\mathrm{IC}_{25}$. All experiments were performed in triplicate.

\section{Results \\ Meso scale discovery (MSD) analysis $S R C$ protein expression}

The level of SRC activation (pSRC) was determined using MSD analysis. In SK-UT-1, treatment with dasatinib resulted in a loss of SRC activation (pSRC/tSRC) at $30 \mathrm{~nm}$ $(16 \% ; \mathrm{p}<0.001), 100 \mathrm{~nm}(8 \% ; \mathrm{p}<0.001)$ and $500 \mathrm{~nm}(2 \%$; $\mathrm{p}<0.001$ ) (Figure 1). The pSRC signal from SK-UT-1 was at least 15 -fold higher than the pSRC signal from the control cells (Additional file 1: Figure S1). In SK-UT-1, there was an increase in tSRC after treatment with singleagent dasatinib at $30 \mathrm{~nm}(148 \%, \mathrm{p}<0.001), 100 \mathrm{~nm}$ $(181 \%, \mathrm{p}<0001)$ and $500 \mathrm{~nm}(172 \%, \mathrm{p}<0.001)$ compared to controls (Additional file 2: Figure S2). In contrast, pSRC levels were significantly decreased after treatment with dasatinib at $30 \mathrm{~nm}(24 \%, \mathrm{p}<0.001), 100 \mathrm{~nm}(14 \%, \mathrm{p}<0.001)$ and $500 \mathrm{~nm}(3 \%, \mathrm{p}<0.001)$ (Additional file 3: Figure S3).

In SK-UT-1B, there was a loss of SRC activation at $30 \mathrm{~nm}(11 \%, \mathrm{p}<0.001), 100 \mathrm{~nm}(11 \%, \mathrm{p}<0.001)$ and $500 \mathrm{~nm}(5 \%, \mathrm{p}<0.001)$ compared to controls (Figure 1). There was a decrease in pSRC levels after treatment with single-agent dasatinib at $30 \mathrm{~nm}(17 \%, \mathrm{p}<0.001), 100 \mathrm{~nm}$ $(7 \%, \mathrm{p}<0.001)$ and $500 \mathrm{~nm}(4 \%, \mathrm{p}<0.001)$ and an increase in tSRC after treatment with single-agent dasatinib at $30 \mathrm{~nm}(152 \%, \mathrm{p}<0.001)$, but a decrease at $100 \mathrm{~nm}(64 \%$, $\mathrm{p}<0.001)$ and $500 \mathrm{~nm}(74 \%, \mathrm{p}<0.001)$ (Additional file 2: Figure S2 and Additional file 3: Figure S3).

\section{Paxillin protein expression}

In SK-UT-1, the ratio of p-paxillin/t-paxillin was unchanged after treatment with dasatinib at $30 \mathrm{~nm}(90 \%, \mathrm{p}<0.001)$ and $100 \mathrm{~nm}(110 \%, \mathrm{p}<0.001)$, but there was a significant reduction at $500 \mathrm{~nm}(16 \%, \mathrm{p}<0.001)$ (Figure 2). There was a significant loss of t-paxillin expression after treatment with dasatinib at $500 \mathrm{~nm}(73 \%, \mathrm{p}=0.01)$, but no change at $30 \mathrm{~nm}(113 \%, \mathrm{p}=0.06)$ and $100 \mathrm{~nm}(92 \%, \mathrm{p}=0.2)$ compared to controls (data not shown). Similar results were observed for p-paxillin levels, with a significant loss after treatment with dasatinib at $500 \mathrm{~nm}(11 \%, \mathrm{p}<0.001)$, but no change at $30 \mathrm{~nm}(100 \%)$ or $100 \mathrm{~nm}(100 \%)$ (data not shown).

In SK-UT-1B, activation of paxillin (p-paxillin/t-paxillin) was significantly inhibited by the presence of dasatinib at $30 \mathrm{~nm}(76 \%), 100 \mathrm{~nm}(1 \%)$ and $500 \mathrm{~nm}$ (3\%) (Figure 2). There was an increase in t-paxillin expression after treatment with dasatinib at $100 \mathrm{~nm}(198 \%, \mathrm{p}<0.001)$, but no change at $30 \mathrm{~nm}(76 \%, \mathrm{p}=0.02)$ or $500 \mathrm{~nm}(93 \%, \mathrm{p}=0.2)$ (data not shown). The expression of p-paxillin was reduced by $42 \%$ after treatment with dasatinib at $30 \mathrm{~nm}(\mathrm{p}<0.001)$ 


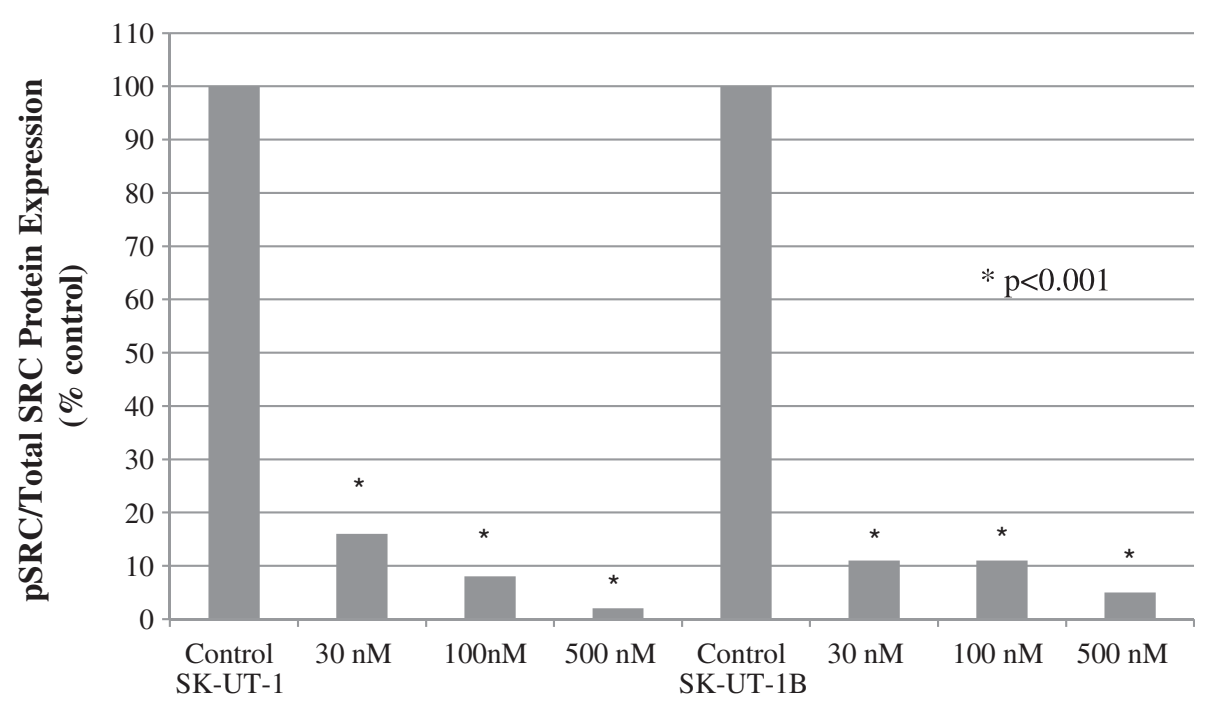

\section{Treatment}

Figure 1 The pSRC/tSRC ratio after treatment with single-agent dasatinib. In SK-UT-1, activation of the SRC kinase (pSRC/tSRC) pathway waS inhibited by dasatinib at $30 \mathrm{~nm}(84 \%), 100 \mathrm{~nm}(92 \%)$ and $500 \mathrm{~nm}(98 \%)$. In SK-UT-1B, the SRC kinase activity was reduced in the presence of dasatinib at $30 \mathrm{~nm}(91 \%), 100 \mathrm{~nm}(91 \%)$ and $500 \mathrm{~nm}(95 \%)$.

and by $97 \%(\mathrm{p}<0.001)$ at 100 and $500 \mathrm{~nm}$ (data not shown).

\section{Anti-proliferative activity of single-agent gemcitabine, docetaxel and dasatinib}

The $\mathrm{IC}_{50}$ for gemcitabine and docetaxel as single agents was calculated for each cell line (Table 1). Growth inhibition with dasatinib was first detected at $100 \mathrm{nmol}$ for
SK-UT-1 (21.7\% inhibition) and for SK-UT-1B (32.8\%). Using increasing concentrations of dasatinib, the maximal growth inhibitory effect of dasatinib for SK-UT-1 was $42.8 \%(1000 \mathrm{~nm})$ and $55.5 \%(4000 \mathrm{~nm})$ for SK-UT-1B (Figure 3). The dasatinib $\mathrm{IC}_{50}$ for SK-UT-1B was $381 \mathrm{nmol} / \mathrm{L}$. Higher concentrations did not achieve greater growth inhibition. In the SK-UT-1 cell line an $\mathrm{IC}_{50}$ was not reached. The $\mathrm{IC}_{25}$ (25\% growth inhibition)

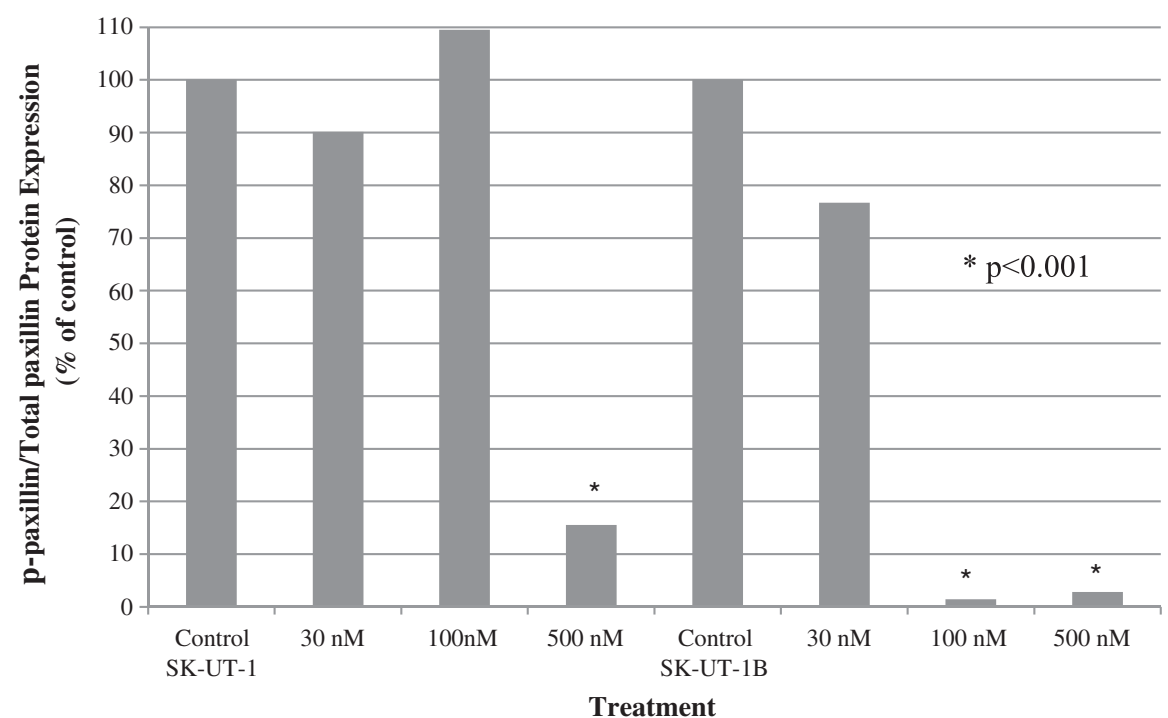

Figure 2 The p-paxillin/t-paxillin ratio after treatment with single-agent dasatinib. Activation of paxillin ( $p$-paxillin/t-paxillin) was inhibited by the presence of dasatinib at $500 \mathrm{~nm}$ in SK-UT-1 (16\%); and $30 \mathrm{~nm}(14 \%), 100 \mathrm{~nm}$ (1\%) and $500 \mathrm{~nm}(3 \%)$ in SK-UT-1B. 
Table 1 IC $_{50}$ of each cytotoxic agent alone and in combination with dasatinib $\left(\mathrm{IC}_{25}\right)$ and coefficient of drug interaction (CDI)

\begin{tabular}{llll}
\hline Cell Line & Drugs & $\mathbf{I C}_{\mathbf{5 0}}$ & CDI \\
\hline SK-UT-1 & & & \\
& Gemcitabine & $4.02(\mathrm{SD} \pm 1.0)$ & \\
& Docetaxel & $4.30(\mathrm{SD} \pm 0.5)$ & \\
& Gemcitabine:Dasatinib $\mathrm{IC}_{25}$ & $2.35(\mathrm{SD} \pm 1.4)$ & 0.72 \\
& Docetaxel:Dasatinib $I C_{25}$ & $1.15(\mathrm{SD} \pm 0.07)$ & 0.80
\end{tabular}

SK-UT-1B

\begin{tabular}{lll} 
Gemcitabine & $2.97(\mathrm{SD} \pm 0.5)$ & \\
Docetaxel & $0.94(\mathrm{SD} \pm 0.6)$ & \\
Gemcitabine:Dasatinib IC 25 & $0.85(\mathrm{SD} \pm 0.6)$ & 0.83 \\
Docetaxel:Dasatinib IC 25 & $0.52(\mathrm{SD} \pm 0.4)$ & 0.93 \\
\hline
\end{tabular}

$\mathrm{SD}=$ standard deviation.

$\mathrm{IC}_{50}=50 \%$ of maximal inhibitory concentration.

$\mathrm{IC}_{25}=25 \%$ of maximal inhibitory concentration.

$\mathrm{CDI}=$ Coefficient of drug interaction. Calculation utilize when one drug does not achieve an $\mathrm{IC}_{50}$.

$\mathrm{CDI}$ scale $=\mathrm{CDI}<1$ indicates synergism between dasatinib and cytotoxic agents, whereas $\mathrm{CDI}<0.7$ indicates significant synergism.

was therefore calculated from dose response curves for each cell line. $\mathrm{The} \mathrm{IC}_{25}$ for dasatinib was $100 \mathrm{~nm}$ for both cell lines.

\section{Drug interaction assessment for combination dasatinib and cytotoxic chemotherapy}

The $\mathrm{IC}_{25}$ of dasatinib calculated for each cell line was used in combination with different concentrations of gemcitabine (e.g. 10, 7.5, 5, 2.5, $1 \mathrm{~nm}$ ) and docetaxel (e.g. 40, 20, 10, 5, $1 \mathrm{~nm}$ ) mixed at a fixed ratio (1:1), respectively. The $\mathrm{IC}_{50}$ obtained from the single agent response curves for both gemcitabine and docetaxel was then compared to the $\mathrm{IC}_{50}$ calculated after adding dasatinib $\left(\mathrm{IC}_{25}\right)$. Results showed that the minimally active dose of dasatinib reduced the $\mathrm{IC}_{50}$ of both gemcitabine and docetaxel for each cell line ranging from 2:1 to $4: 1$ (2-4 fold inhibition) (Table 1) (Figure 4).

For SK-UT-1 and SK-UT-1B, the combination of gemcitabine-dasatinib at all concentrations analyzed demonstrated decreased cell viability (Figure 5A and E) and yielded a CDI $<1$ indicating synergistic effects (average CDI $=0.72$ and 0.83 for SK-UT-1 and SK-UT-1B, respectively) (Figure 5B and F). Similarly, in both cell lines the combination of docetaxel-dasatinib demonstrated decreased cell viability (Figure 5C and G) and synergistic effects (average CDI $=0.80$ and 0.93 for SK-UT-1 and SK-UT-1B, respectively) (Figure $5 \mathrm{D}$ and $\mathrm{H}$ ).

The median effect analysis for combining gemcitabine and docetaxel revealed synergistic effect in SK-UT-1 $(\mathrm{CI}=0.59)$ and moderate antagonism in SK-UT-1B $(\mathrm{CI}=1.36)$ (Table 2). The results of the simultaneous and sequential drug experiments using dasatinib $\mathrm{IC}_{25}$, gemcitabine and docetaxel in a triple-drug combination are shown in Table 2. The greatest anti-proliferative activity (synergistic) in SK-UT-1 was seen with the simultaneous triple-drug combination of dasatinib, gemcitabine and docetaxel $(\mathrm{CI}=0.46)$. The remainder of the sequential triple-drug combinations revealed a moderate antagonistic effect $(\mathrm{CI}=1.07-1.8)$ (Table 2). In SK-UT-1B, the simultaneous triple-drug combination of gemcitabine, docetaxel and dasatinib revealed moderate antagonistic effect (anti-proliferative effect $(\mathrm{CI}=1.4)$ ). Sequential dasatinib followed by combination gemcitabine and docetaxel as well as gemcitabine followed by combination docetaxel and dasatinib yielded synergistic effects ( $\mathrm{CI}=0.9$ for both). In contrast, combination dasatinib and docetaxel followed by gemcitabine demonstrated an additive effect $(\mathrm{CI}=1.0)$. The remaining sequential combinations produced an antagonistic effect (CI ranging from 1.21-1.5) (Table 2).

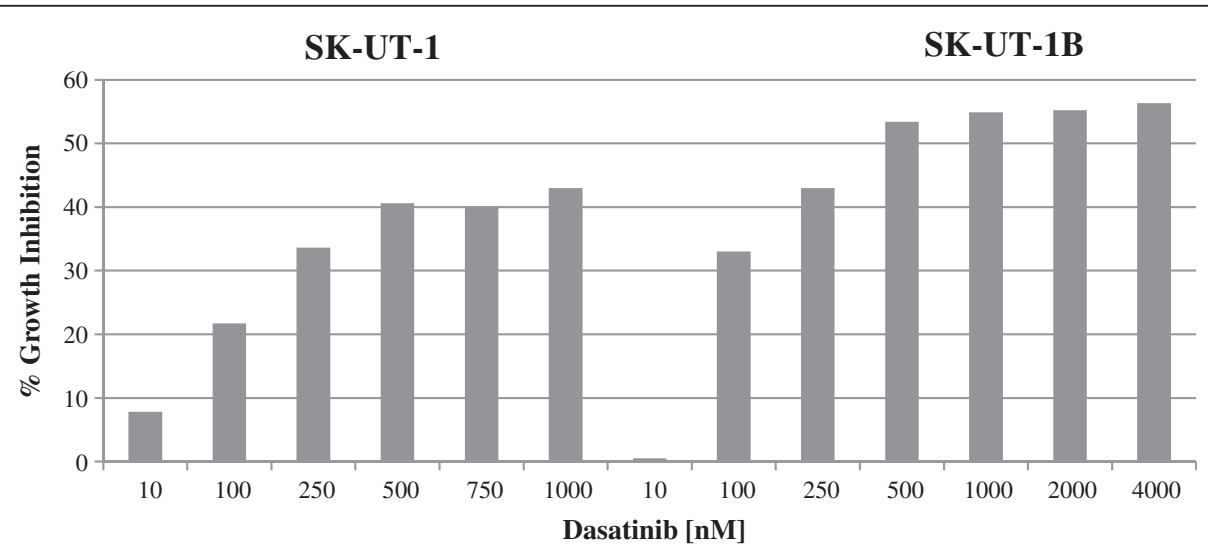

Figure 3 Growth inhibition assay. Dasatinib showed minimal to modest activity against leiomyosarcoma cell lines. Maximal growth inhibitory effect for SK-UT-1 and SK-UT-1B was $42.8 \%$ and 55\% respectively. 
A.

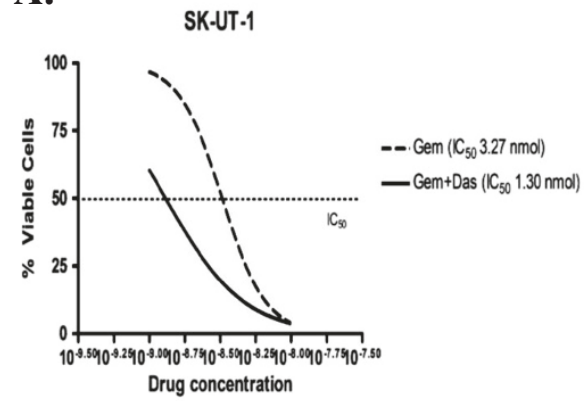

C.

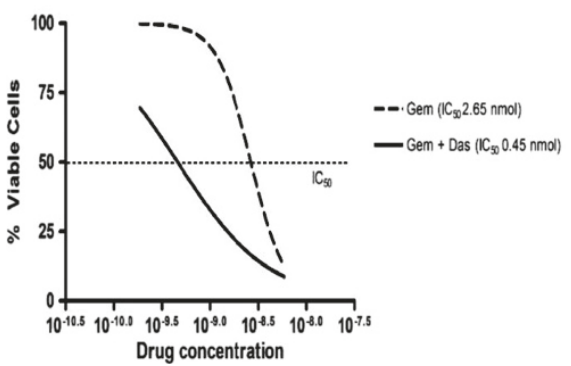

B.

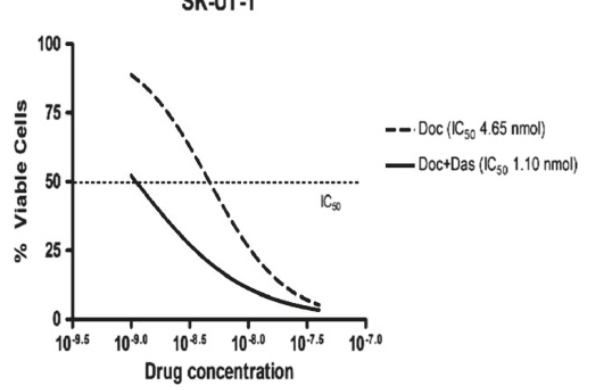

D.

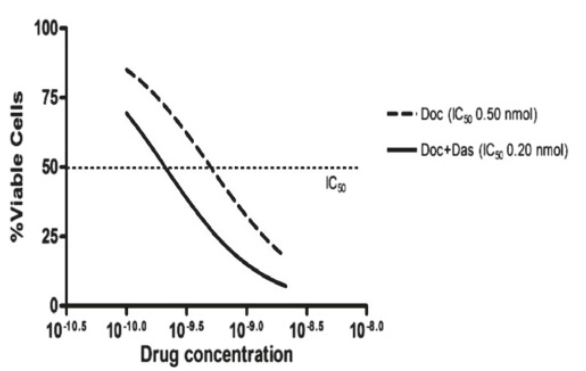

Figure $4 \mathrm{IC}_{50}$ obtained from single agent response curves for gemcitabine and docetaxel was compared to the IC 50 obtained after adding dasatinib at a minimally active concentration $\left(\mathbf{I C}_{\mathbf{2 5}}\right)$. Graphics above represents the results of a single experiment for SK-UT-1 (A \& B) and SK-UT-1B (C \& D). (Gem = gemcitabine; Das = dasatinib; Doc = docetaxel).

\section{Discussion and conclusion}

Dasatinib has not been shown to be a potent agent when used alone in a variety of solid tumors [14]. Schrage et al. previously reported their findings of dasatinib in chondrosarcoma cell lines [15]. The maximum percent of growth inhibition in the chondrosarcoma cell lines was approximately $50 \%$. They also explored dasatinib's effect on SRC phosphorylation and caspase-3- mediated apoptosis. Although dasatinib treatment of chondrosarcoma decreased SRC phosphorylation, indicating target inhibition, it did not result in an increase in apoptosis. Therefore, the inhibition of SRC and its family of kinases was not sufficient to promote cell death. In a large clinical trial of patients with incurable sarcoma, which included 47 participants with LMS, dasatinib did not have a significant anti-tumor activity as a single agent [16]. Based on pre-clinical and clinical data, it appears that single agent dasatinib does not have significant clinical activity in soft tissue sarcomas.

However, our pre-clinical study investigating the activity of dasatinib in combination with gemcitabine and docetaxel in uterine LMS demonstrates that dasatinib acts synergistically with gemcitabine or docetaxel in a two-drug combination and in select triplet combinations in the analyzed uterine LMS cell lines. Our findings in sequential and triple combination yielded conflicting results. Interestingly, the simultaneous triple-drug combination of dasatinib $\mathrm{IC}_{25}$, gemcitabine and docetaxel in SK-UT-1 yielded a synergistic effect, although the magnitude of that effect is probably minimal $(\mathrm{CI}=0.59$ to 0.46$)$. Conversely, the gemcitabine and docetaxel doublet demonstrated an antagonistic effect in SK-UT-1B cells and that effect was not reversed with the addition of dasabinib in the simultaneous triple-drug combination. However, in select sequencing experiments, dasatinib enhanced the anti-proliferative effect of gemcitabine and docetaxel; gemcitabine + docetaxel + dasatinib; dasatinib followed by docetaxel + gemcitabine; and gemcitabine followed by docetaxel + dasatinib. In the latter two experiments, this finding may be due to avoidance of the antagonistic interaction of the gemcitabine and docetaxel doublet in the SK-UT-1B cell line as well as partially attributed to the synergistic effect of the dasatinib doublet. The results with the sequential dasatinib followed by combination gemcitabine and docetaxel indicate a possible role for priming with dasatinib.

We hypothesize that our synergistic findings may in part be due to the inhibition of the SRC pathway. Our MSD analysis revealed that dasatinib inhibited the SRC pathway based on reduction of pSRC and p-paxillin protein expression and ratios $\mathrm{pSRC/tSRC}$ and p-paxcillin/ $\mathrm{t}$-paxillin. Even at very low doses of dasatinib $(30 \mathrm{nM})$, the SRC pathway was inhibited in both uLMS cell lines. This suggests that an optimal biologic effect on SRC can occur with low doses of dasatinib. However, expression of paxillin was only inhibited at high doses. A possible 

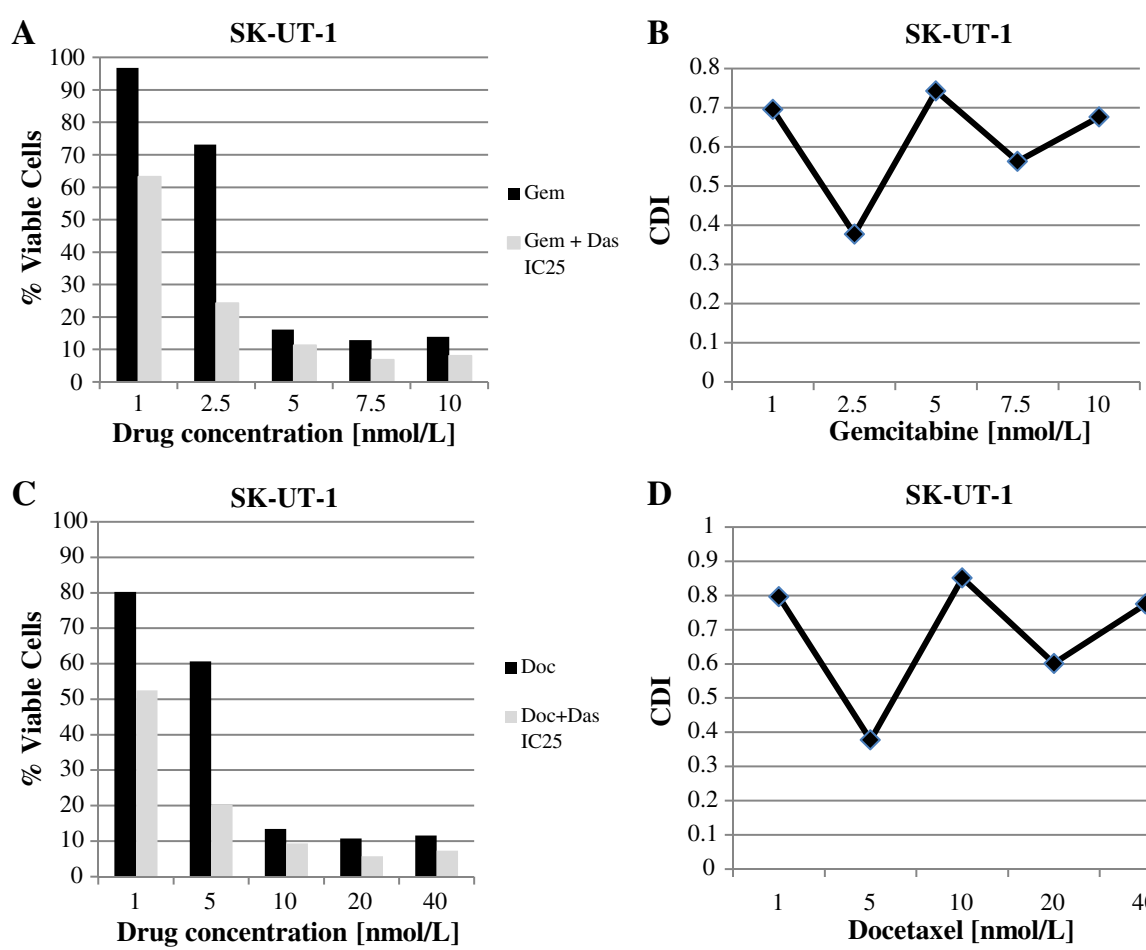

D

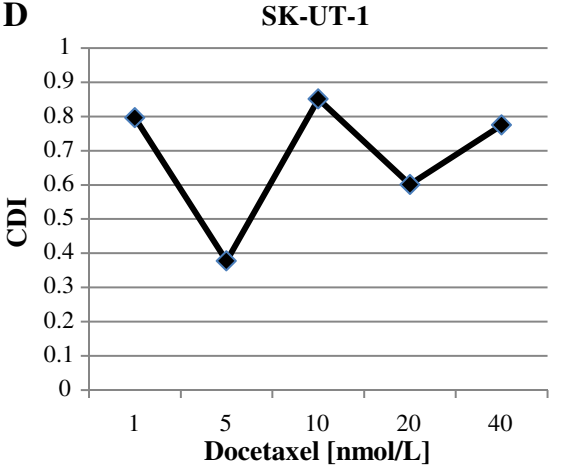

E

SK-UT-1B

F

SK-UT-1B
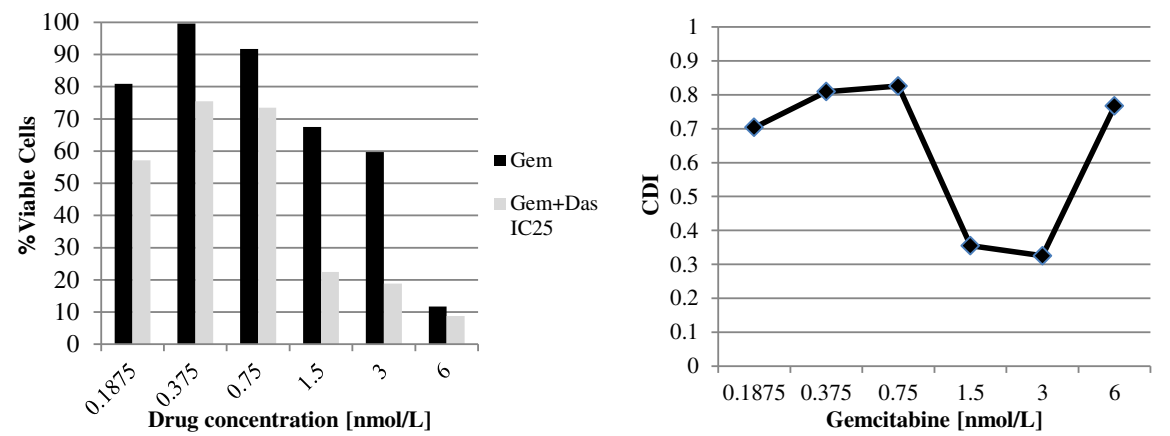

G

SK-UT-1B

H

SK-UT-1B
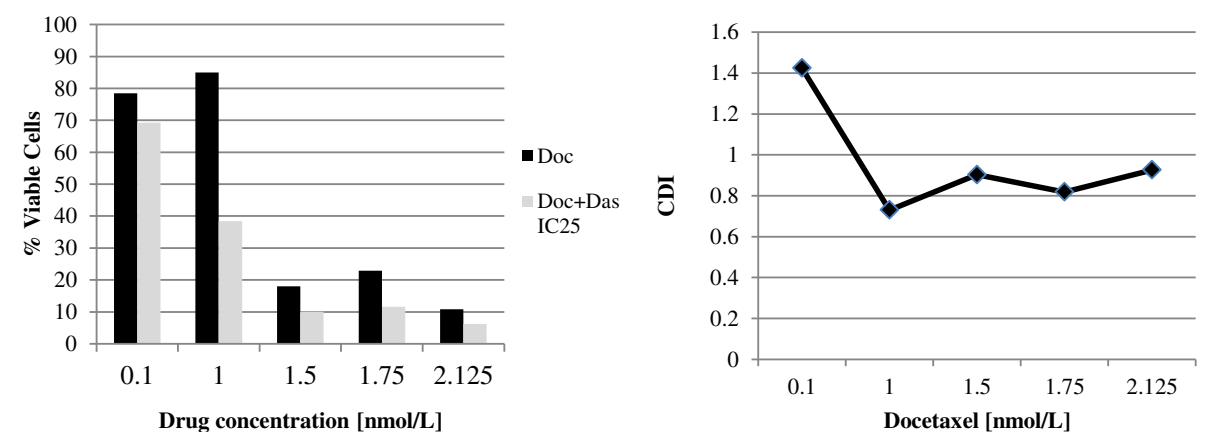

Figure 5 The inhibitory effect of gemcitabine and docetaxel at different concentrations combined with dasatinib $\mathrm{IC}_{25}$ and the coefficient of drug interaction (CDI). $C D I<1$ indicates synergism, $C D I<0.7$ significant synergism, $C D I=1$ additivity and $C D>1$ antagonism. In SK-UT-1, the combination of gemcitabine-dasatinib, at all concentrations analyzed demonstrated decreased cell viability (A) and yielded a CDI $<1$ indicating synergistic effects (average $C D I=0.72)(B) ;$ the combination of docetaxel-dasatinib demonstrated decreased cell viability (C) and synergistic effects (average $\mathrm{CDI}=0.80)(\mathbf{D})$. In SK-UT-1B the combination of gemcitabine-dasatinib, at all concentrations analyzed demonstrated decreased cell viability $(\mathbf{E})$ and yielded a $C D I<1$ indicating synergistic effects (average $C D I=0.83)(\mathbf{F})$; the combination of docetaxel-dasatinib demonstrated decreased cell viability $(\mathbf{G})$ and synergistic effects $(C D I=0.93)(\mathbf{H})$. 
Table 2 The combination index $(\mathrm{CI})$ in uterine LMS cell lines

\begin{tabular}{lll}
\hline Cell Line & Drugs & $\begin{array}{l}\text { Combination index } \\
(\mathbf{C l}) \text { at the } \text { IC }_{\mathbf{5 0}}\end{array}$ \\
\hline SK-UT-1 & & \\
& Gemcitabine:Docetaxel & 0.59 \\
& Gemcitabine + Docetaxel + Dasatinib & 0.46 \\
& Dasatinib- > Gemcitabine + Docetaxel & 1.77 \\
& Gemcitabine + Docetaxel- > Dasatinib & 1.14 \\
& Dasatinib + Gemcitabine- > Docetaxel & 1.32 \\
& Dasatinib + Docetaxel- > Gemcitabine & 1.07 \\
Gemcitabine- > Docetaxel + Dasatinib & 1.8 \\
Docetaxel- > Gemcitabine + Dasatinib & $\mathrm{N} / \mathrm{D}$
\end{tabular}

SK-UT-1B

$\begin{array}{ll}\text { Gemcitabine:Docetaxel } & 1.36 \\ \text { Gemcitabine + Docetaxel + Dasatinib } & 1.4 \\ \text { Dasatinib- > Gemcitabine + Docetaxel } & 0.9 \\ \text { Gemcitabine + Docetaxel- > Dasatinib } & 1.36 \\ \text { Dasatinib + Gemcitabine- > Docetaxel } & 1.21 \\ \text { Dasatinib + Docetaxel- > Gemcitabine } & 1.0 \\ \text { Gemcitabine- > Docetaxel + Dasatinib } & 0.9 \\ \text { Docetaxel- > Gemcitabine + Dasatinib } & 1.5\end{array}$

Combination index scale: $\mathrm{Cl}<0.9$ synergistic, $\mathrm{Cl}=0.9-1.1$ additive, $\mathrm{Cl}=1.1-1.2$ slight antagonism,

$\mathrm{Cl}=1.2-1.45$ moderate antagonism, $\mathrm{Cl}=1.45-3.3$ antagonism, $\mathrm{Cl}=3.3-10$ strong antagonism.

$\mathrm{N} / \mathrm{D}=$ not determined.

explanation for this is that downstream substrates of SRC pathway may be activated via different pathways. The SRC pathway is a complex pathway with convergent and divergent interactions. It is not clear that the anti-proliferative effect we noted in our study was a direct result of inhibition of the SRC pathway. Most recently, Shank et al. showed that EGFR, VEGF and especially c-KIT were expressed in uterine LMS specimens [9]. In particular, $57 \%$ of uterine LMS specimens expressed c-KIT. Therefore, dasatinib's anti-proliferative effect seen in our study may be via the inhibition of the c-KIT pathway in addition to or instead of inhibition of the SRC pathway.

Another important property of dasatinib not related to cytotoxicity, lies in its ability to inhibit migration and cell invasion. Dasatinib has been shown to inhibit cell migration in non-small cell lung cancer, head and neck squamous cell cancer, neuroblastoma, and multiple soft tissue and bone sarcoma cell lines $[8,17,18]$. Johnson and colleagues reported that the anti-migratory effects of dasatinib were present regardless of the effects seen on proliferation and survival [17]. In addition, Shor et al. showed that dasatinib significantly inhibited cellular invasion using matrigel invasion chambers. With the exception of one rhabdomyosarcoma cell line, the $\mathrm{IC}_{50}$ dose to inhibit cell migration and invasion in the soft tissue sarcoma cell lines ranged from $4-65 \mathrm{nmol} / \mathrm{L}$ and was similar to the $\mathrm{IC}_{50}$ dose (range $3 \mathrm{nmol} / \mathrm{L}$ to $68 \mathrm{nmol} / \mathrm{L}$ ) to inhibit SRC phosphorylation [8]. This finding suggests that SRC inhibition may be responsible for suppression of sarcoma cellular migration and invasion. The universal effects seen on migration and invasion suggest that beneficial clinical effects may be achieved without direct cancer cell cytotoxicity and provides a possible role of dasatinib in preventing metastasis. Inhibition of metastasis is especially important in aggressive tumors such as LMS which are known to rapidly spread to distant sites.

While we and others have reported synergistic and additive effects with dasatinib and a variety of chemotherapeutic agents in preclinical studies of ovarian and breast cancer cell lines and ovarian cancer xenografts [19-21], the clinical activity of dasatinib either alone or in combination with cytotoxic agents in solid tumors has been disappointing. In a phase I trial of dasatinib in combination with paclitaxel and carboplatin in patients with advanced or recurrent ovarian cancer, the triplet combination demonstrated clinical activity but was also associated with a higher than expected rate of hematologic toxicity. Pharmacokinetic analysis showed that concurrent administration of dasatinib with paclitaxel did not significantly alter either dasatinib or paclitaxel drug concentrations [14]. Two randomized studies of another SRC inhibitor, saracatinib, with chemotherapy in ovarian cancer demonstrated no improvement in response rate or survival, but higher toxicity $[22,23]$. Therefore, the addition of dasatinib to chemotherapy is unlikely to be beneficial in an unselected patient population and there is a need to identify biomarkers that can be used to direct therapy. Of note, these studies used the maximum tolerated dose (MTD). In our data, we observed an increased antiproliferative effect of dasatinib in combination with cytotoxic agents at a minimal active dose. Hence, combination effects may be beneficial at lower doses of dasatinib in the treatment of uterine leiomyosarcoma.

Up-regulation of the $S R C$ pathway has been shown to be associated with resistance to cytotoxic therapy. In mucinous ovarian cancer cell lines, Matsuo et al. revealed that treatment with oxaliplatin induced phosphorylation of SRC kinase and this contributed to the chemoresistance observed in this tumor. This induced activity was subsequently inhibited by concurrent administration of dasatinib, which resulted in a synergistic anti-tumor effect [24].

To our knowledge, there are no clinical trials that have evaluated the toxicity of gemcitabine and docetaxel in combination with dasatinib. In a prospective trial of women with completely resected uterine leiomyosarcoma, treatment with gemcitabine plus docetaxel was generally well-tolerated. 
Potential serious toxicities associated with gemcitabine plus docetaxel in the advanced disease setting included pulmonary toxicity and myelosuppression requiring administration of granulocyte-colony stimulating factor [3]. Based on our prior experience, we anticipate that the addition of dasatinib to gemcitabine and docetaxel would be associated with even greater hematologic toxicity. A phase I evaluation would be required to determine if biologically relevant doses could be delivered with the triplet combination. However, the disparate sequential combination results in our cell lines was not expected and requires further study. Alternatively, doublet therapy with dasatinib plus gemcitabine or docetaxel may be more effective than triplet therapy.

We acknowledge the limitations of our study, which include the use of only two LMS cell lines rather than primary tumor, and the use of in vitro models. Further investigation in in vivo models is warranted to confirm our in vitro results. Further study is also needed to determine if dasatinib exerts its function via inhibition of the SRC pathway or via other tyrosine kinases, such as BCR-ABL, c-KIT, EPHA2, EGFR and PDGF, or a combination. In summary, we are the first to report an anti-proliferative effect of dasatinib in combination with cytotoxic agents in uterine leiomyosarcoma.

\section{Additional files}

Additional file 1: Figure S1. Expression of phospho SRC (pSRC) in the SK-UT-1 cell line. "KDR" are vascular endothelial growth factor cells use as control group. Compare to this group, both SKUT-1 and SKUT-1B demonstrated a much higher PSRC signal.

Additional file 2: Figure S2. The total SRC protein expression after treatment with single agent dasatinib in SK-UT-1 and SK-UT-1B cell lines. In SK-UT-1, there was an increase in tSRC after treatment with single-agent dasatinib at $30 \mathrm{~nm}(148 \%, \mathrm{p}<0.001), 100 \mathrm{~nm}(181 \%, \mathrm{p}<0001)$ and $500 \mathrm{~nm}(172 \%, \mathrm{p}<0.001)$ compared to controls. In SK-UT-1B, there was an increase in $\mathrm{SRRC}$ after treatment with single-agent dasatinib at 30 $\mathrm{nm}(152 \%, \mathrm{p}<0.001)$, but a decrease at $100 \mathrm{~nm}(64 \%, \mathrm{p}<0.001)$ and 500 $\mathrm{nm}(74 \%, p<0.001)$

Additional file 3: Figure S3. pSRC protein expression after treatment with single agent dasatinib in SK-UT-1 and SK-UT-1B cell lines. In SK-UT-1, pSRC levels were significantly decreased after treatment with dasatinib at $30 \mathrm{~nm}(24 \%, p<0.001), 100 \mathrm{~nm}(14 \%, p<0.001)$ and $500 \mathrm{~nm}$ $(3 \%, p<0.001)$. In SK-UT-1B, there was a decrease in pSRC levels after treatment with single-agent dasatinib at $30 \mathrm{~nm}(17 \%, p<0.001), 100 \mathrm{~nm}$ $(7 \%, p<0.001)$ and $500 \mathrm{~nm}(4 \%, \mathrm{p}<0.001)$.

\section{Competing interest}

The authors declare that they have no competing interests to be disclosed.

\section{Authors' contributions}

AAS, DT, DJA and MLA provided substantial contributions to conception and design, acquisition, interpretation of data, drafting the article and final approval of the version to be published. LG and RW were responsible for supervising proper laboratory techniques and assure compliance with established protocols. They actively participated in all laboratories task including: growth of cell cultures, mixing chemotherapy agents in investigation with cell lines and recording results. They provided substantial contributions to drafting the article and final approval of the version to be published. JJ and ABN performed all immunogenicity assays and provided substantial contributions to drafting the article and final approval of the version to be published. All authors agree to be accountable for all aspects of the work in ensuring that questions related to the accuracy or integrity of any part of the work are appropriately investigated and resolved.

\section{Acknowledgments}

We thank the Charles Hammond Research Fund and the Sandra H. Barbeau Foundation for granting financial assistance to conduct this project the National Cancer Institute for providing the cytotoxic agents.

\section{Author details}

'Division of Gynecologic Oncology, Duke Cancer Institute, Durham, NC 27710, USA. 'Division of Gynecologic Oncology, University of Minnesota, Minneapolis, MN 55455, USA. ${ }^{3}$ Department of Medicine, Duke University Medical Center, Durham, NC 27710, USA. ${ }^{4}$ East Carolina University School of Medicine, Greenville, NC 27834, USA. ${ }^{5}$ DUMC 3079, Gynecologic Oncology, Duke University Medical Center, Durham, NC 27710, USA.

Received: 22 March 2014 Accepted: 2 July 2014

Published: 30 September 2014

\section{References}

1. Nordal RR, Thoresen SO: Uterine sarcomas in Norway 1956-1992: incidence, survival and mortality. Eur J Cancer 1997, 33(6):907-11.

2. Lurain J, Piver M: Uterine sarcomas: clinical features and management In Gynecological Oncology. Edited by Coppleson M. Edinburgh; London; Melbourne: Churchill Livingstone: 1992:827-42.

3. Hensley ML, Ishill N, Soslow R, Larkin J, Abu-Rustum N, Sabbatini P, Konner J, Tew W, Spriggs D, Aghajanian CA: Adjuvant gemcitabine plus docetaxel for completely resected stages I-IV high grade uterine leiomyosarcoma: Results of a prospective study. Gynecol Oncol 2009, 112(3):563-7.

4. Hensley ML, Maki R, Venkatraman E, Geller G, Lovegren M, Aghajanian C, Sabbatini P, Tong W, Barakat R, Spriggs DR: Gemcitabine and docetaxel in patients with unresectable leiomyosarcoma: results of a phase II trial. J Clin Oncol 2002, 20(12):2824-31.

5. Luo FR, Barrett YC, Yang Z, Camuso A, McGlinchey K, Wen ML, Smykla R, Fager K, Wild R, Palme H, Galbraith S, Blackwood-Chirchir A, Lee FY: Identification and validation of phospho-SRC, a novel and potential pharmacodynamic biomarker for dasatinib (SPRYCEL), a multi-targeted kinase inhibitor. Cancer Chemother Pharmacol 2008, 62(6):1065-74.

6. Lombardo L, Lee FY, Chen P, Norris D, Barrish JC, Behnia K, Castaneda S, Cornelius LA, Das J, Doweyko AM, Fairchild C, Hunt JT, Inigo I, Johnston K, Kamath A, Kan D, Klei H, Marathe P, Pang S, Peterson R, Pitt S,

Schieven GL, Schmidt RJ, Tokarski J, Wen ML, Wityak J, Borzilleri RM: Discovery of N-(2-chloro-6-methyl- phenyl)-2-(6-(4-(2-hydroxyethyl)piperazin-1-yl)-2-methylpyrimidin-4- ylamino)thiazole-5-carboxamide (BMS-354825), a dual Src/Abl kinase inhibitor with potent antitumor activity in preclinical assays. J Med Chem 2004, 47(27):6658-61.

7. Weiner TM, Liu ET, Craven RJ, Cance WG: Expression of growth factor receptors, the focal adhesion kinase, and other tyrosine kinases in human soft tissue tumors. Ann Surg Oncol 1994, 1(1):18-27.

8. Shor AC, Keschman EA, Lee FY, Muro-Cacho C, Letson GD, Trent JC: Dasatinib inhibits migration and invasion in diverse human sarcoma cell lines and induces apoptosis in bone sarcoma cells dependent on SRC kinase for survival. Cancer Res 2007, 67(6):2800-8.

9. Shank J, Frisch N, Rhode J, Liu J: Identification of Molecular Markers for Targeted Treatment of Uterine Sarcomas. Gynecol Oncol 2013, 131(1):282.

10. Cao SS, Zhen YS: Potentiation of antimetabolite antitumor activity in vivo by dipyridamole and amphotericin B. Cancer Chemother Pharmacol 1989, 24(3):181-6.

11. Xu SP, Sun GP, Shen YX, Peng WR, Wang H, Wei W: Synergistic effect of combining paeonol and cisplatin on apoptotic induction of human hepatoma cell lines. Acta Pharmacol Sin 2007, 28(6):869-78.

12. Chou TC: Theoretical basis, experimental design, and computerized simulation of synergism and antagonism in drug combination studies. Pharmacol Rev 2006, 58(3):621-81.

13. Chou TC, Talalay P: Quantitative analysis of dose-effect relationships: the combined effects of multiple drugs or enzyme inhibitors. Adv Enzyme Regul 1984, 22:27-55.

14. Secord AA, Teoh DK, Barry WT, Yu M, Broadwater G, Havrilesky LJ, Lee PS, Berchuck A, Lancaster J, Wenham RM: A phase I trial of dasatinib, an SRC-family kinase inhibitor, in combination with paclitaxel and 
carboplatin in patients with advanced or recurrent ovarian cancer. Clin Cancer Res 2012, 18(19):5489-98.

15. Schrage YM, Briaire-de Bruijn IH, de Miranda NF, van Oosterwijk J, Taminiau AH, van Wezel T, Hogendoorn PC, Bovée JV: Kinome profiling of chondrosarcoma reveals SRC-pathway activity and dasatinib as option for treatment. Cancer Res 2009, 69(15):6216-22.

16. Schuetze S, Wathen K, Choy E, Samuels BL, Ganjoo KN, Staddon AP, von Mehren M, Chow WA, Trent JC, Baker LH: Results of a Sarcoma Alliance for Research through Collaboration (SARC) phase II trial of dasatinib in previously treated, high-grade, advanced sarcoma. J Clin Oncol 2010, 28(suppl; abstr 10009):15s.

17. Johnson FM, Saigal B, Talpaz M, Donato NJ: Dasatinib (BMS-354825) tyrosine kinase inhibitor suppresses invasion and induces cell cycle arrest and apoptosis of head and neck squamous cell carcinoma and non-small cell lung cancer cells. Clin Cancer Res 2005, 11(19 Pt 1):6924-32.

18. Timeus F, Crescenzio N, Fandi A, Doria A, Foglia L, Cordero di Montezemolo L: In vitro antiproliferative and antimigratory activity of dasatinib in neuroblastoma and Ewing sarcoma cell lines. Oncol Rep 2008, 19(2):353-9.

19. Teoh D, Ayeni TA, Rubatt JM, Adams DJ, Grace L, Starr MD, Barry WT, Berchuck A, Murphy SK, Secord AA: Dasatinib (BMS-35482) has synergistic activity with paclitaxel and carboplatin in ovarian cancer cells. Gynecol Oncol 2011, 121(1):187-92.

20. Chen T, Pengetnze Y, Taylor CC: Src inhibition enhances paclitaxel cytotoxicity in ovarian cancer cells by caspase-9-independent activation of caspase-3. Mol Cancer Ther 2005, 4(2):217-24.

21. Company BMS: Preclinical pharmacology of dasatinib, a SRC protein kinase inhibitor. 2003, Control No. 930003300.

22. Poole C, Lisyanskaya A, Rodenhuis S, Kristensen G, Pujade-Lauraine E, Cantarini M, Emeribe U, Stuart M, Ray Cl: A randomized phase II clinical trial of the SRC inhibitor saracatinib (AZD0530) and carboplatin 1 paclitaxel (C1P) versus C1P in patients with advanced platinumsensitive epithelial ovarian cancer. Ann Oncol 2010, 21(Suppl 8):S313.

23. McNeish IA, Ledermann JA, Webber LC, James LE, Kaye SB, Rustin GJS, Hall G, Clamp A, Earl HM, Banerjee SN, Kristeleit RS, Raja F, Feeney A, Lawrence C, Dawson-Athey L, Persic M, Khan I: A Randomized Placebo-Controlled Trial of Saracatinib (AZD0530) Plus Weekly Paclitaxel in Platinum-Resistant Ovarian, Fallopian-Tube, Or Primary Peritoneal Cancer (SaPPrOC). Chicago, IL: American Society of Clinical Oncology annual meeting; 2013.

24. Matsuo K, Nishimura M, Bottsford-Miller JN, Huang J, Komurov K, ArmaizPena GN, Shahzad MM, Stone RL, Roh JW, Sanguino AM, Lu C, Im DD, Rosenshien NB, Sakakibara A, Nagano T, Yamasaki M, Enomoto T, Kimura T, Ram PT, Schmeler KM, Gallick GE, Wong KK, Frumovitz M, Sood AK: Targeting SRC in mucinous ovarian carcinoma. Clin Cancer Res 2011, 17(16):5367-78

doi:10.1186/2053-6844-1-2

Cite this article as: Lopez-Acevedo et al:: Dasatinib (BMS-35482) potentiates the activity of gemcitabine and docetaxel in uterine leiomyosarcoma cell lines. Gynecologic Oncology Research and Practice 2014 1:2

\section{Submit your next manuscript to BioMed Central and take full advantage of:}

- Convenient online submission

- Thorough peer review

- No space constraints or color figure charges

- Immediate publication on acceptance

- Inclusion in PubMed, CAS, Scopus and Google Scholar

- Research which is freely available for redistribution 